\title{
CINmetrics: An R package for chromosomal instability analysis
}

\author{
Vishal H. Oza ${ }^{1,}$, Jennifer L. Fisher ${ }^{1}$, Roshan Darji ${ }^{1}$, Brittany N. Lasseigne ${ }^{1}$ \\ 1 Department of Cell, Developmental and Integrative Biology, Heersink \\ School of Medicine, University of Alabama at Birmingham, Birmingham, \\ 35294, USA
}

\section{*vishoza@uab.edu}

\begin{abstract}
Genomic instability has been an important hallmark in cancer and more recently in neurodegenrative diseases. Chromosomal instability, as a measure of genomic instability, has been used to characterize clinical and biological phenotypes associated with these diseases by measuring structural and numerical chromosomal alterations. There have been multiple Chromosomal Instability Scores developed across many studies in the literature; however, these scores have not been compared because of a lack of single tool available to calculate these various metrics. Here, we provide an $\mathrm{R}$ package CINmetrics, that calculates six different chromosomal instability scores and allows direct comparison between them. We also show how these scores differ by applying CINmetrics to breast cancer data from The Cancer Genome Atlas (TCGA).

Availability: The package is available on CRAN at https://cran.r-project.org/package=CINmetrics and on github at https://github.com/lasseignelab/CINmetrics
\end{abstract}

\section{Introduction}

Genomic instability, one of the hallmarks of cancer, is measured in many forms such as chromosomal instability, microsatellite instability, and instability characterized by increased frequency of base-pair mutations (Bakhoum and Cantley, 2018; Pikor et al. 2013 ; Negrini et al. 2010). Particularly, chromosomal instability (CIN) is associated with cancer progression, tumor immunity, and inflammation (Pikor et al. 2013 , Bach et al. 2019). Recently, CIN has been shown to contribute to other diseases than cancer, including neurodegenerative diseases (Hou et al., 2017; Yurov et al., 2019).

Chromosomal instability is broadly defined as the change in number and structure of chromosomes (Vargas-Rondón et al. 2017 ). CIN has been measured directly from tumour specimens by capturing errors during anaphase segregation or indirectly by measuring numerical and structural chromosomal alterations across cell populations. There have been various CIN scores developed across multiple studies involving different cancers which calculate numerical and structural alterations in the chromosome (McGranahan et al. 2012). The differences in calculation of these scores have been associated with different clinical and biological phenotype (Baumbusch et al., 2013 , Davison et al., 2014, Roylance et al., 2011, Bonnet et al., 2012); however, there has been no systematic comparison of different CIN scores and how they vary across and within 
different cancers. The primary reason being lack of availability of computational framework to calculate these CIN scores. While other packages are available to calculate chromosomal instability (Song et al., 2017), they are limited to a single chromosomal instability score and do not provide a framework to calculate and compare other CIN scores. Here, we provide an $\mathrm{R}$ package that provides a unified framework to calculate multiple CIN metrics on same dataset. This package will accelerate chromosomal instability studies by facilitating score comparisons across cancers or other diseases.

\section{Materials and Methods}

The chromosomal instability metrics were mined from the cancer literature and implemented as functions in our CINmetrics R package. The six functions (tai, taiModified, cna, countingBreakPoints, countingBaseSegments, fga) are outlined below based on the similarity of the algorithms used to calculate them.

\subsection{Total aberration index (tai) and Modified Total Aberration Index (taiModified)}

Total Aberration Index (TAI) was proposed by (Baumbusch et al., 2013$)$ to measure the genomic aberrations in serous ovarian cancers. TAI calculates absolute area under the curve for a copy number segment profile generated by piecewise constant fitting (PCF) algorithm. Biologically, TAI can be interpreted as absolute deviation from the normal copy number state averaged over all genomic locations. TAI provides a numerical measure in terms of both prevalence as well as the genomic size of copy number variations in tumors. One of the limitations of TAI is that since it was designed for studying advance stage ovarian tumors and short aberrations found in the early tumors have low impact on TAI. Therefore, TAI should be used to study global scale genomic disorganization that most likely occur in late stage tumors.

tai implemented in CINmetrics takes into account only those sample values that are in aberrant copy number state, i.e. has a mean segment values of less than or equal to -0.2 and greater than or equal to +0.2 .

$$
\text { Total Aberration Index }=\frac{\sum_{i=1}^{R} d_{i} \cdot y_{S_{i}}}{\sum_{i=1}^{R} d_{i}}
$$

where $y_{S_{i}} \leq-0.2$ and $y_{S_{i}} \geq+0.2$ represents the mean segment value. Alternatively, taiModfied takes into account all the mean segment values and thus preserves the "directionality" of the score.

$$
\text { Modified Total Aberration Index }=\frac{\sum_{i=1}^{R} d_{i} \cdot y_{S_{i}}}{\sum_{i=1}^{R} d_{i}}
$$

where $y_{S_{i}}$ represents the mean segment value

\subsection{Copy number abnormality (cna) and Counting number of break points (countingBreakPoints)}

Copy Number Abnormality (CNA) was developed by (Davison et al., 2014 ) for studying aneuploidy in superficial gastroesophageal adenocarcinoma. An individual CNA is defined as the segment with copy number outside the predefined range of 1.7 to 2.3 with the score of 2 indicating no loss or gain (assuming that the tumor is diploid) as determined by Partek segmentation algorithm. Total CNA for the sample can thus be defined as total number of individual CNAs. CNA can be thought of as a measure of 
segmental aneuploidy. cna implemented in CINmetrics is similar except we define individual CNA as the segment with copy number less than equal to -0.2 and greater than equal to +0.2 with segment mean of 0 indicating no loss or gain. We chose \pm 0.2 as a conservative cutoff for TCGA data as described in (Laddha et al., 2014). The users can modify the cutoff by modifying segmentMean parameter.

$$
\text { Total Copy Number Abnormality }=\sum_{i=1}^{R} n_{i}
$$

where $n_{i}$ represents number of segments with $y_{S_{i}} \leq-0.2$ and $y_{S_{i}} \geq+0.2$, and the minimum segment length $d_{i}$ is greater than or equal to 10 .

countingBreakPoints is similar to the total breakpoints implemented in (Lee et al. 2011). Segments with mean less than equal to -0.2 and greater than equal to +0.2 and having number of probes above the user defined threshold, are counted and then the value is doubled to account for 3' and 5' ends. The measure gives similar results to cna

$$
\text { Number of Break Points }=\sum_{i=1}^{R}\left(n_{i} \cdot 2\right)
$$

where $n_{i}$ represents number of segments with $y_{S_{i}} \leq-0.2$ and $y_{S_{i}} \geq+0.2$

\subsection{Counting altered base segments (countingBaseSegments)and Fraction of the genome altered ( $f g a)$}

Counting altered base segments and fraction of the genome altered are modified implementation of the Genome Instability Index (GII) as described in (Chin et al. 2007). The GII was computed in two different ways, both based on calculating common regions of alteration (CRA) and both approaches showed high concordance.

$$
\text { Number of Altered Bases }=\sum_{i=1}^{R} d_{i}
$$

where $d_{i}$ represents length of segments with $y_{S_{i}} \leq-0.2$ and $y_{S_{i}} \geq+0.2$

fga implemented in our package is based on identifying common regions of alterations as fraction of the genome altered. Therefore, the $f g a$ values are normalized by dividing it by the length of the genome covered. countingBaseSegments on the other hand calculates the common regions of alteration.

$$
\text { Fraction Genome Altered }=\frac{\sum_{i=1}^{R} d_{i}}{G}
$$

where $d_{i}$ represents length of segments with $y_{S_{i}} \leq-0.2$ and $y_{S_{i}} \geq+0.2$ and $G$ represents genome length covered. The default value is calculated based on length of each probe on Affeymetrix 6.0 array and excludes the sex chromosomes. One important difference to note is that in the original GII calculations, the algorithm merges the non-overlapping regions between samples, whereas fga and countingBaseSegements implemented in CINmetrics package do not merge the non-overlapping regions.

\section{Application}

We used harmonized masked copy number segment data for breast cancer (BRCA) from The Cancer Genome Atlas (Cancer Genome Atlas Network, 2012, Cerami et al., 2012), 
A

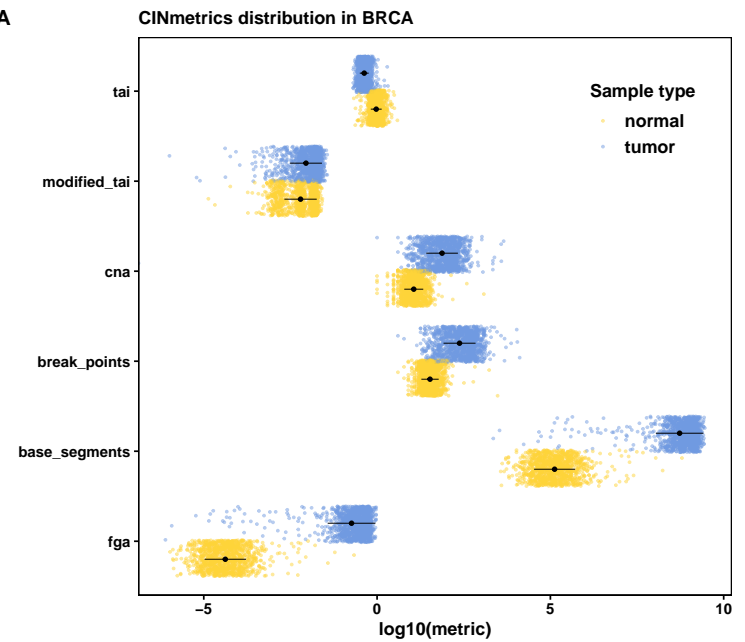

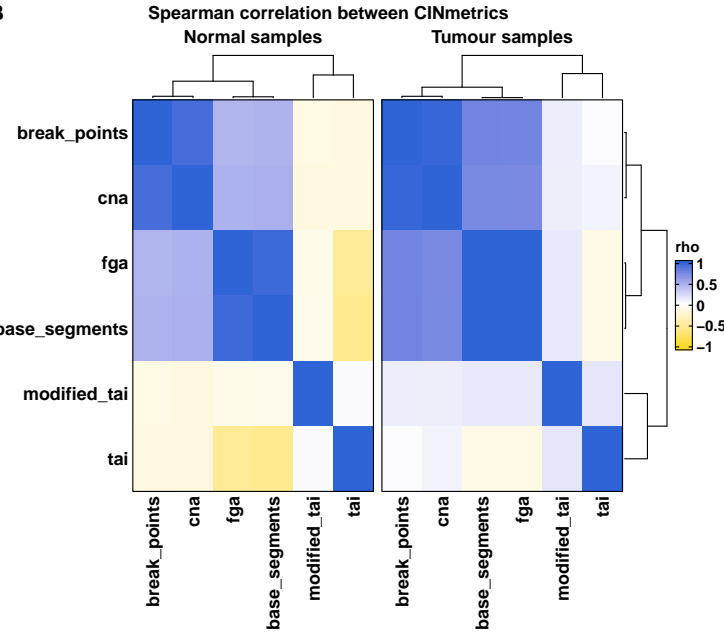

Figure 1. CINmetrics applied to BRCA dataset from TCGA, A) shows the distribution of metrics between normal (yellow) and tumour(blue) samples, the black dot indicates the mean and the black line indicates the standard deviation B) shows the heatmap of the spearman correlation and complete linkage clustering of the metrics in normal and tumour samples.

to visualize and compare the chromosomal instability metrics implemented in CINmetrics package. We chose the breast cancer data as it has been shown to exhibit chromosomal instability and thus provides a good framework for applying CINmetrics e.g. (Duijf et al., 2019, Voutsadakis, 2021). Figure 1A shows the distribution of CINmetrics in BRCA data for normal and tumour samples. The metrics have been $\log _{10}$ scaled to allow for comparison between them. cna, countingBreakPoints, fga, and countingBaseSegments show an overall pattern of increased genomic instability in tumour samples compared to normal. However, the difference in mean and standard deviation between the two classes (normal and tumour) is very different between these metrics. tai and taiModified do not capture this global pattern of difference between normal and tumour samples. As mentioned earlier, tai and taiModified are best suited for late stage cancers, thus should be used as a measure for studying overall genomic disorganization in individual patients with advanced tumours and not as a measure of genomic instability comparison between normal and tumour samples.

To further understand and characterize the relationship between various metrics implemented in CINmetrics, we performed Spearman correlation (Spearman, 1904), followed by complete linkage clustering (Vijaya et al. 2019) as shown in Figure 1B. The clustering further demonstrated that cna, countingBreakPoints, fga, and

countingBaseSegments are more similar and therefore highly correlated compared to tai and taiModified. Furthermore, this relationship is preserved in both normal and tumour samples indicating the four metrics show consistent results and can be used for comparing genomic instability between the two.

\section{Conclusion}

CIN has been one of the important factors in understanding disease etiology and progression in cancer (Pikor et al. 2013, Bach et al. 2019) and is becoming increasingly important for others like neurodegenerative diseases (Hou et al., 2017, Yurov et al., 2019). Numerous methods have been developed to quantitate and characterize the role of chromosomal instability in specific cancers, however, lack of comprehensive tool that 
calculates these metrics has limited direct comparison between them. Here, we have collected chromosomal instability metrics from the literature and provide them as an $\mathrm{R}$ package and associated vignette that allows for reproducible calculations and comparisons. We used BRCA data from The Cancer Genome Atlas to show how the metrics relate to each other. Further studies are needed to better characterize and understand these metrics, for e.g. what kind of instabilities drive each of these metrics and whether combining more than one metric gives a better idea about the genomic instability in cancer. This package will provide a useful framework to perform such studies.

\section{Acknowledgments}

We would like to thank members of the Lasseigne Lab for testing the CINmetrics package including Tabea M. Soelter.

\section{Funding}

This work has been supported by the NIH R00HG009678 (to B. Lasseigne), NIH R00HG009678-04S1 (to B. Lasseigne), UAB AMC21 (to J. Fisher), and UAB Startup Fund Support (to B.Lasseigne).

\section{References}

Bach, D.-H., Zhang, W., and Sood, A. K. (2019). Chromosomal instability in tumor initiation and development. Cancer Res., 79(16), 3995-4002.

Bakhoum, S. F. and Cantley, L. C. (2018). The multifaceted role of chromosomal instability in cancer and its microenvironment. Cell, 174(6), 1347-1360.

Baumbusch, L. O., Helland, Å., Wang, Y., Liestøl, K., Schaner, M. E., Holm, R., Etemadmoghadam, D., Alsop, K., Brown, P., Australian Ovarian Cancer Study Group, Mitchell, G., Fereday, S., DeFazio, A., Bowtell, D. D. L., Kristensen, G. B., Lingjærde, O. C., and Børresen-Dale, A.-L. (2013). High levels of genomic aberrations in serous ovarian cancers are associated with better survival. PLoS One, 8(1), e54356.

Bonnet, F., Guedj, M., Jones, N., Sfar, S., Brouste, V., Elarouci, N., Banneau, G., Orsetti, B., Primois, C., de Lara, C. T., Debled, M., de Mascarel, I., Theillet, C., Sévenet, N., de Reynies, A., MacGrogan, G. and Longy, M. (2012). An array CGH based genomic instability index (G2I) is predictive of clinical outcome in breast cancer and reveals a subset of tumors without lymph node involvement but with poor prognosis. BMC Med. Genomics, 5, 54.

Cancer Genome Atlas Network (2012). Comprehensive molecular portraits of human breast tumours. Nature, 490(7418), 61-70.

Cerami, E., Gao, J., Dogrusoz, U., Gross, B. E., Sumer, S. O., Aksoy, B. A., Jacobsen, A., Byrne, C. J., Heuer, M. L., Larsson, E., Antipin, Y., Reva, B., Goldberg, A. P., Sander, C., and Schultz, N. (2012). The cbio cancer genomics portal: an open platform for exploring multidimensional cancer genomics data. Cancer Discov., 2(5), 401-404.

Chin, S. F., Teschendorff, A. E., Marioni, J. C., Wang, Y., Barbosa-Morais, N. L., Thorne, N. P., Costa, J. L., Pinder, S. E., van de Wiel, M. A., Green, A. R., Ellis, I. O., Porter, P. L., Tavaré, S., Brenton, J. D., Ylstra, B., and Caldas, C. (2007). High-resolution aCGH and expression profiling identifies a novel genomic subtype of ER negative breast cancer. Genome Biol., 8(10), R215.

Davison, J. M., Yee, M., Krill-Burger, J. M., Lyons-Weiler, M. A., Kelly, L. A., Sciulli, C. M., Nason, K. S., Luketich, J. D., Michalopoulos, G. K., and LaFramboise, W. A. (2014). The degree of segmental aneuploidy measured by total copy number abnormalities predicts survival and recurrence in superficial gastroesophageal adenocarcinoma. PLoS One, 9(1), e79079.

Duijf, P. H. G., Nanayakkara, D., Nones, K., Srihari, S., Kalimutho, M., and Khanna, K. K. (2019). Mechanisms of genomic instability in breast cancer. Trends Mol. Med., 25(7), 595-611.

Hou, Y., Song, H., Croteau, D. L., Akbari, M., and Bohr, V. A. (2017). Genome instability in alzheimer disease. Mech. Ageing Dev., 161(Pt A), 83-94. 
Laddha, S. V., Ganesan, S., Chan, C. S., and White, E. (2014). Mutational landscape of the essential autophagy gene BECN1 in human cancers. Mol. Cancer Res., 12(4), 485-490.

Lee, A. J. X., Endesfelder, D., Rowan, A. J., Walther, A., Birkbak, N. J., Futreal, P. A., Downward, J., Szallasi, Z., Tomlinson, I. P. M., Howell, M., Kschischo, M., and Swanton, C. (2011). Chromosomal instability confers intrinsic multidrug resistance. Cancer Res., 71(5), 1858-1870.

McGranahan, N., Burrell, R. A., Endesfelder, D., Novelli, M. R., and Swanton, C. (2012). Cancer chromosomal instability: therapeutic and diagnostic challenges. EMBO Rep., 13(6), 528-538.

Negrini, S., Gorgoulis, V. G., and Halazonetis, T. D. (2010). Genomic instability-an evolving hallmark of cancer. Nat. Rev. Mol. Cell Biol., 11(3), 220-228.

Pikor, L., Thu, K., Vucic, E., and Lam, W. (2013). The detection and implication of genome instability in cancer. Cancer Metastasis Rev., 32(3-4), 341-352.

Roylance, R., Endesfelder, D., Gorman, P., Burrell, R. A., Sander, J., Tomlinson, I., Hanby, A. M., Speirs, V., Richardson, A. L., Birkbak, N. J., Eklund, A. C., Downward, J., Kschischo, M., Szallasi, Z., and Swanton, C. (2011). Relationship of extreme chromosomal instability with long-term survival in a retrospective analysis of primary breast cancer. Cancer Epidemiol. Biomarkers Prev., 20(10), $2183-2194$.

Song, L., Bhuvaneshwar, K., Wang, Y., Feng, Y., Shih, I.-M., Madhavan, S., and Gusev, Y. (2017). CINdex: A bioconductor package for analysis of chromosome instability in DNA copy number data. Cancer Inform., 16, 1176935117746637.

Spearman, C. (1904). nthe proof and measurement of association between two things, oamerican J.

Vargas-Rondón, N., Villegas, V. E., and Rondón-Lagos, M. (2017). The role of chromosomal instability in cancer and therapeutic responses. Cancers, $\mathbf{1 0}(1)$.

Vijaya, Sharma, S., and Batra, N. (2019). Comparative study of single linkage, complete linkage, and ward method of agglomerative clustering. In 2019 International Conference on Machine Learning, Big Data, Cloud and Parallel Computing (COMITCon), pages 568-573.

Voutsadakis, I. A. (2021). The landscape of chromosome instability in breast cancers and associations with the tumor mutation burden: An analysis of data from TCGA. Cancer Invest., 39(1), 25-38.

Yurov, Y. B., Vorsanova, S. G., and Iourov, I. Y. (2019). Chromosome instability in the neurodegenerating brain. Front. Genet., 10, 892 . 